\title{
Deep Learning-Based Ultrasonic Testing to Evaluate the Porosity of Additively Manufactured Parts with Rough Surfaces
}

\author{
Seong-Hyun Park ${ }^{1}$, Jung-Yean Hong ${ }^{1} \mathbb{D}$, Taeho Ha ${ }^{2}$, Sungho Choi ${ }^{3, *}$ and Kyung-Young Jhang ${ }^{4, *(D)}$ \\ 1 Department of Mechanical Convergence Engineering, Hanyang University, Seoul 04763, Korea; \\ seonghyun@hanyang.ac.kr (S.-H.P.); hongjy127@hanyang.ac.kr (J.-Y.H.) \\ 2 Department of 3D Printing, Korea Institute of Machinery \& Materials, Daejeon 34103, Korea; \\ taehoha@kimm.re.kr \\ 3 Department of Flexible and Printable Electronics, LANL-JBNU Engineering Institute-Korea, \\ Jeonbuk National University, Jeonju-si 54896, Korea \\ 4 School of Mechanical Engineering, Hanyang University, Seoul 04763, Korea \\ * Correspondence: schoi@jbnu.ac.kr (S.C.); kyjhang@hanyang.ac.kr (K.-Y.J.); Tel.: +82-63-219-5437 (S.C.); \\ +82-2-2220-4220 (K.-Y.J.)
}

Citation: Park, S.-H.; Hong, J.-Y.; Ha,

T.; Choi, S.; Jhang, K.-Y. Deep

Learning-Based Ultrasonic Testing to

Evaluate the Porosity of Additively

Manufactured Parts with Rough

Surfaces. Metals 2021, 11, 290.

https: / / doi.org/10.3390/met11020290

Academic Editor: Atila Ertas

Received: 20 December 2020

Accepted: 10 January 2021

Published: 8 February 2021

Publisher's Note: MDPI stays neutral with regard to jurisdictional claims in published maps and institutional affiliations.

Copyright: (C) 2021 by the authors. Licensee MDPI, Basel, Switzerland. This article is an open access article distributed under the terms and conditions of the Creative Commons Attribution (CC BY) license (https:/ / creativecommons.org/licenses/by/ $4.0 /)$.

\begin{abstract}
Ultrasonic testing (UT) has been actively studied to evaluate the porosity of additively manufactured parts. Currently, ultrasonic measurements of as-deposited parts with a rough surface remain problematic because the surface lowers the signal-to-noise ratio (SNR) of ultrasonic signals, which degrades the UT performance. In this study, various deep learning (DL) techniques that can effectively extract the features of defects, even from signals with a low SNR, were applied to UT, and their performance in terms of the porosity evaluation of additively manufactured parts with rough surfaces was investigated. Experimentally, the effects of the processing conditions of additive manufacturing on the resulting porosity were first analyzed using both optical and scanning acoustic microscopy. Second, convolutional neural network (CNN), deep neural network, and multi-layer perceptron models were trained using time-domain ultrasonic signals obtained from additively manufactured specimens with various levels of porosity and surface roughness. The experimental results showed that all the models could evaluate porosity accurately, even that of the as-deposited specimens. In particular, the $\mathrm{CNN}$ delivered the best performance at $94.5 \%$. However, conventional UT could not be applied because of the low SNR. The generalization performance when using newly manufactured as-deposited specimens was high at $90 \%$.
\end{abstract}

Keywords: additive manufacturing; porosity; rough surface; ultrasonic testing; convolutional neural network; deep neural network; multi-layer perceptron

\section{Introduction}

Additive manufacturing (AM) is the process of depositing materials, layer upon layer, to create an object from a 3D computer-aided design [1-4]. The distinctive advantages of AM are that it can be used to produce innovative, complex designs and the fact that it is lightweight compared to conventional subtractive or casting manufacturing. Owing to these advantages, this manufacturing method has been actively studied in various fields [5-7]. A current major concern is manufacturing defects that can occur in the interior of AM parts and their effects on the integrity of these parts [8,9]. Porosity, defined as air-filled cavities inside a material, is a typical manufacturing defect found in AM parts, and is the result of deviation from the optimal AM processing conditions. Because porosity can severely aggravate the mechanical properties of AM parts, it has been of significant interest to researchers to evaluate the extent of porosity in manufactured parts [10-12].

Ultrasonic testing (UT) is a well-known non-destructive testing method and can be used to effectively evaluate the porosity [11-13] and properties of a material, including its strength, elastic modulus, and material density [14-16]. Previous studies have reported 
that an ultrasonic wave propagating through a porous medium is scattered and delayed so that the wave velocity decreases $[17,18]$. The relation between ultrasonic velocity and the amount of porosity has been studied [13]. Studies on UT for porosity evaluation in AM parts have also been reported. Slotwinski et al. [19] experimentally investigated the correlation between the porosity content and ultrasonic velocity in AM parts using a contact ultrasonic method. The relation between the ultrasonic velocity and attenuation, and the properties of microstructures, including the porosity and grain size, was studied by Karthik et al. [20], who used a water-immersion ultrasonic method. Javidrad et al. [21] correlated the elastic modulus affected by the porosity content with the ultrasonic velocity in varying the propagation directions of ultrasonic waves using a contact ultrasonic method.

Although the effectiveness of UT in evaluating the porosity of AM parts has previously been verified, as-deposited AM parts with rough surfaces still present a major problem in UT. Ultrasonic signals obtained from a rough surface have a low signal-to-noise ratio (SNR), which degrades the UT performance. To overcome this problem, previous studies commonly used various surface polishing methods as a post-processing step to prepare the surface of as-deposited AM parts whose roughness exceeded a certain level. However, this post-processing step often resulted in an increase in the overall time and cost of the AM process [22-25]. The aforementioned discussion highlights the need to develop an innovative UT that can evaluate porosity without surface polishing, that is, even under rough surface conditions.

Recently, deep learning (DL) methods have been actively employed in various fields, including speech recognition [26], computer vision [27], and signal processing [28,29]. Other approaches have involved the application of DL to UT for feature extraction in defect detection [30,31]. One advantage thereof is the excellent ability of DL to consistently and accurately interpret ultrasonic signals for the characterization and detection of defects, compared with conventional UT that are error-prone and often depend on the experience and skills of non-destructive inspectors [32,33]. In addition, DL is able to effectively extract and defect features, even from ultrasonic signals with a low SNR [34,35].

Although DL has been used in UT in many studies for the detection of cracks [36,37], corrosion [38], welding defects [39,40], and others [31,33-35,41], only a few studies on DLbased UT under low SNR conditions have been reported. For example, Munir et al. [34] used DL for the classification of welding defects at low SNRs. They measured ultrasonic signals from welding defects and then added various levels of artificial noise. They reported that their convolutional neural network $(\mathrm{CNN})$ outperformed the fully connected deep neural network (DNN) and multi-layer perceptron (MLP) as the SNR decreased. Zhang et al. [42] investigated DL to diagnose bearing faults at low SNRs. They added various levels of artificial noise to bearing fault vibration signals measured by an acoustic emission method. Similar to the aforementioned study, the classification performance of $\mathrm{CNN}$ was more accurate than that of DNN, MLP, and a support vector machine at low SNRs. However, to the best of the authors' knowledge, studies on DL-based UT to inspect AM parts have not been reported. Apart from this, it would be difficult to directly apply the results of previous studies to DL-based UT at low SNRs for the inspection of AM parts. This is because the reduction of the SNR of ultrasonic signals as a result of the surface roughness of AM parts is more severe than that due to the artificial noise used in previous studies.

In this study, we investigated the performance of DL-based UT to evaluate the porosity of AM parts with a rough surface. Several surface polishing methods were used after the $\mathrm{AM}$ process to obtain AM specimens with different levels of surface roughness. The porosity content of AM specimens was controlled by varying the AM processing conditions. The experimental procedures were as follows. (1) The amount of porosity was quantitatively determined and the porosity generation mechanisms were evaluated using scanning acoustic microscopy (SAM) and optical microscopy (OM), respectively. (2) The training and testing datasets were composed of time-domain ultrasonic signals acquired by measuring AM specimens, which were divided into 10 classes and labeled according to their porosity content. CNN, DNN, and MLP models were used and trained using these datasets. The 
testing performance of the three models was evaluated and compared with respect to the surface roughness. Furthermore, the applicability of the conventional UT was also considered for performance comparison. (3) The generalization performance was evaluated using newly manufactured AM specimens that were not used to train the DL model in order to verify the generalizability of the pre-trained model.

\section{Experiments}

\subsection{Fabrication of Porosity-Induced Specimens with Different Levels of Surface Roughness}

Ten AM specimens with various levels of porosity were created using a commercial selective laser melting machine (SLM 280 2.0, SLM Solutions, Lübeck, Germany) and commercially pure Ti powder with a particle diameter range of 20-63 $\mu \mathrm{m}$. The specimens were $20 \mathrm{~mm} \times 10 \mathrm{~mm} \times 3 \mathrm{~mm}$ in size and were numbered from $\# 1$ to \#10. The porosity content was controlled by varying the AM processing parameters, such as the laser power and laser scanning speed, which are known as the main indices that affect the porosity content of AM specimens. The processing parameters were selected after several preliminary tests and are summarized in Table 1. Generally, increasing the laser power or decreasing the scanning speed causes over-melting porosity. The opposite situation gives rise to porosity with a lack of fusion (LOF) [43]. Recent studies have found that porosity may also develop differently as a result of the variation in other properties such as the conduction-keyhole mode conversion of the melt pool [44], laser absorptivity [45], energy dissipation rate, and interaction time [46]. A detailed analysis of porosity mechanisms is provided in the next section. After specimen fabrication, both sides of the surfaces were polished to obtain the "smooth condition" using wire electrical discharge machining (EDM), where the arithmetic mean roughness $\left(R_{a}\right)$ measured by a general roughness tester was $0.65 \mu \mathrm{m}$.

Table 1. Laser power and laser scanning speed used for specimen creation.

\begin{tabular}{|c|c|c|c|c|c|c|c|c|c|c|}
\hline $\begin{array}{l}\text { Specimen } \\
\text { Name }\end{array}$ & $\# 1$ & $\# 2$ & \#3 & $\# 4$ & $\# 5$ & $\# 6$ & \#7 & $\# 8$ & \#9 & $\# 10$ \\
\hline Laser power $(\mathrm{W})$ & 275 & 355 & 275 & 235 & 315 & 355 & 355 & 355 & 235 & 235 \\
\hline Scanning speed $(\mathrm{mm} / \mathrm{s})$ & 1091 & 1315 & 849 & 1187 & 1458 & 1517 & 1644 & 1793 & 768 & 725 \\
\hline
\end{tabular}

To consider different surface roughness conditions, we fabricated 10 additional AM specimens, numbered from $\# 1^{\prime}$ to $\# 10^{\prime}$, the surfaces of which on both sides had different degrees of roughness. The surface on one side was polished to attain the "medium condition" by a general hand grinder that was used to separate specimens from the baseplate of a 3D printer. The other side was "rough condition", which corresponded to the as-deposited raw surface. The $R_{a}$ of each surface was $3.1 \mu \mathrm{m}$ and $6.4 \mu \mathrm{m}$, respectively, which are also listed in Table 2 together with those of the \#1-\#10 specimens. Except for the roughness conditions, all the other properties were the same as \#1-\#10 specimens. A photograph of the AM specimens is shown in Figure 1. Only the porosity of the specimens with smooth surfaces was examined with SAM. The training/testing datasets were constructed by using all three surface conditions.

Table 2. Three different conditions of surface roughness used in the experiments.

\begin{tabular}{ccc}
\hline Roughness Condition & Ra Value $(\mu \mathrm{m})$ & Surface Polishing Method \\
\hline Smooth condition & 0.65 & Wire electrical discharge machining \\
Medium condition & 3.1 & Hand grinder \\
Rough condition & 6.4 & X (As-deposited state) \\
\hline
\end{tabular}




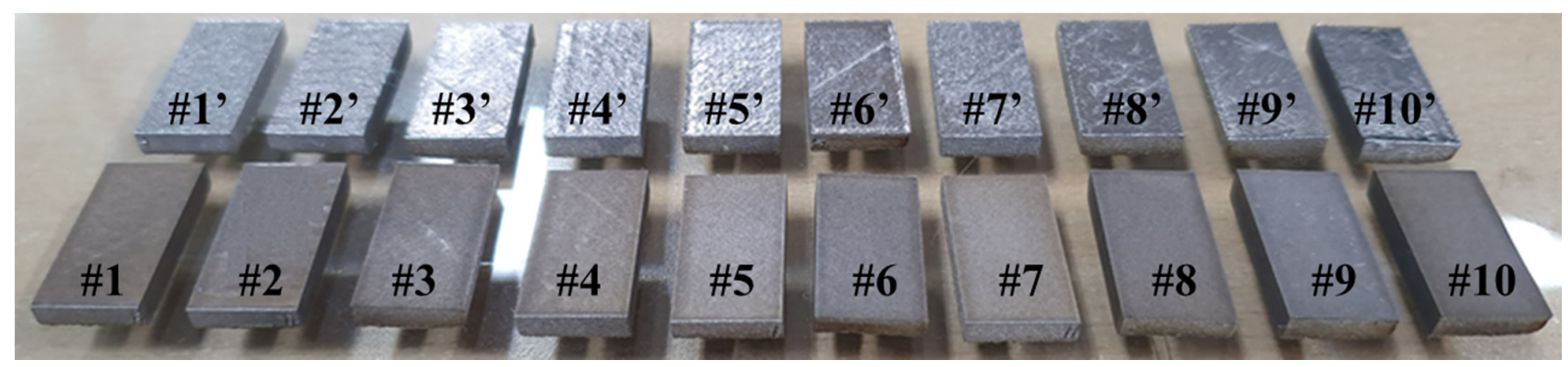

Figure 1. Photographic image of the additive manufacturing (AM) specimens.

\subsection{Porosity Examination}

SAM was used to quantify the porosity content of the AM specimens. C-mode imaging using a scanning acoustic microscope (HS-1000, Sonix, Springfield, VA, USA) was conducted with a $75 \mathrm{MHz}$ focusing-type transducer. Only AM specimens finished to obtain the "smooth condition" were tested because the scanning quality of this imaging is highly influenced by the surface condition of specimens [47]. The focal point and the C-mode window were positioned at the center of the specimen. The window size was set to $1.5 \mathrm{~mm}$, which corresponded to half the thickness of the specimen, to obtain comprehensive results for porosity. After the SAM analysis, a general optical microscope (GX53, OLYMPUS, Inc., Olympus, Tokyo, Japan) was used to analyze the shape and type of porosity. For the OM analysis, the surfaces of AM specimens with the "smooth condition" were additionally polished to lower their surface roughness to $0.04 \mu \mathrm{m}$.

Figure 2 shows the $\mathrm{C}$-mode images that were obtained. The images clearly show that the porosities, represented as bright spots, are distributed differently depending on the AM processing conditions. These $\mathrm{C}$-mode images were used to quantify the amount of porosity by using the open-source software Image [48]. Based on the $6 \mathrm{~dB}$ drop method, each image was subjected to the binarization process, and the calculated porosity contents, defined as the ratio of the pore area to the total area in two-dimensional space, are summarized in Table 3. Note that these porosity contents are relative values [48]. According to the amount of porosity, they were labeled from "Porosity level 1" to "Porosity level 10". The measured porosity content increases as the specimen number increases. Because specimen \#1 was manufactured under the optimal processing conditions, its porosity content was the lowest at $0.7 \%$ as determined by SAM. Almost no porosity was observed in the OM image shown in Figure 3a. Under this condition, the volume laser energy input (LEI) in the melt pool was $70 \mathrm{~J} / \mathrm{mm}^{3}$. The porosity contents of specimens \#2 and \#3 were in the range of $2.5-7.5 \%$. The LEI of these specimens was 75 and $90 \mathrm{~J} / \mathrm{mm}^{3}$, respectively, which was within the over-melting condition; this causes not only welded particles and wavy surfaces but also entrapped gas, resulting in small pits and gas porosity, as shown in Figure $3 b$. Specimens \#4-\#8 were manufactured under LEI conditions of $55-65 \mathrm{~J} / \mathrm{mm}^{3}$. The porosity content was within the range 7.5-27.5\%, slightly higher than those of \#2 and \#3. Generally, more porosity is created under LOF conditions than under over-melting conditions [49]. The lack of LEI prevents the powder in the inter- and intra-layers from melting sufficiently, which results in LOF porosity with un-melted powder, as shown in Figure 3c. Although these specimens had similar LEI levels, more pores were observed in specimens \#7 and \#8 than in \#4-\#6, as shown in Figure 2. This may be due to the insufficient interaction time of the laser to melt the powder owing to the higher scanning speed [49]. The LEI of specimens \#9 and \#10 was 85-90 J/ $\mathrm{mm}^{3}$, within the over-melting condition. Despite the LEI being similar to that of \#2 and \#3, the porosity content was significantly higher (over $27.5 \%$ ). The reason may be a combination of high LEI and low laser power, in which case a shallow melt pool is generated, which may not be able to penetrate the previously deposited layers. This result may yield a large number of pits with un-melted powder between the interlayers, with the result that these specimens have the highest porosity, as shown in Figure 3d. 

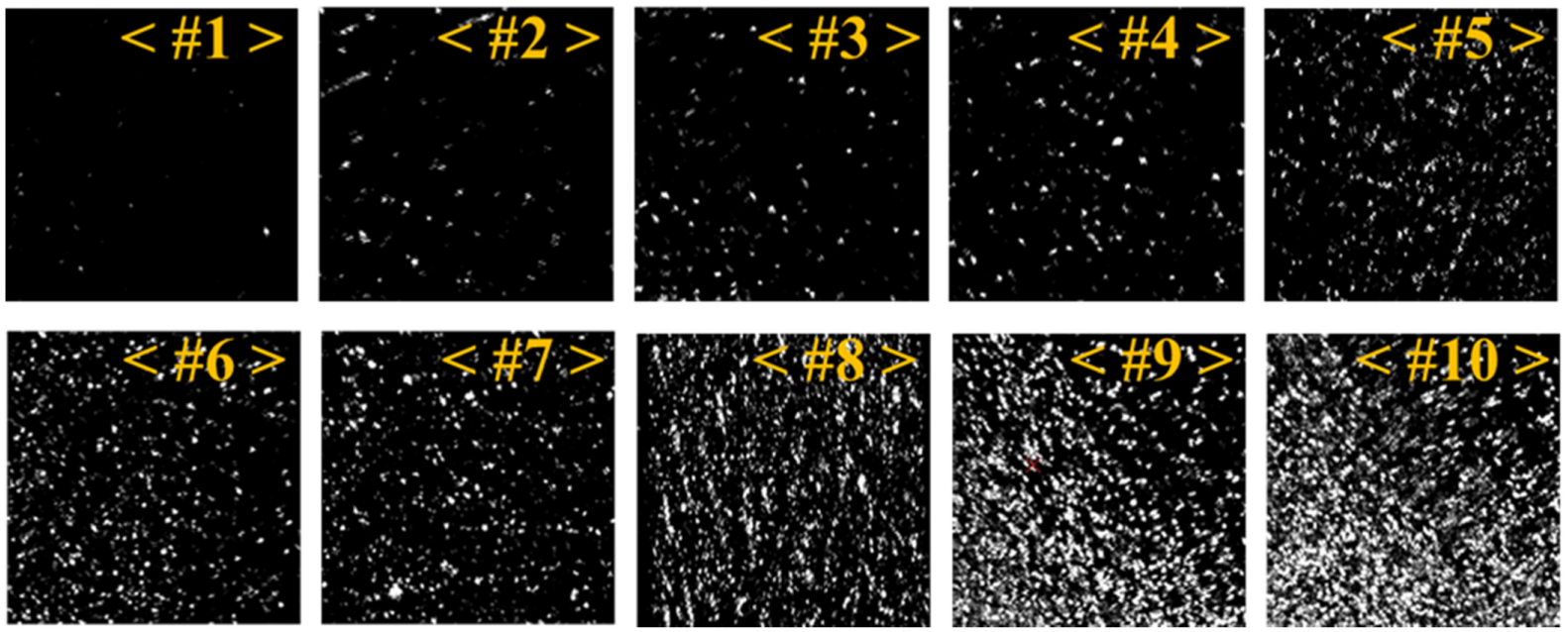

Figure 2. C-scan images obtained from specimens \#1 to \#10.

Table 3. Porosity content of specimens \#1 to \#10 as measured using scanning acoustic microscopy (SAM), and the corresponding porosity levels.

\begin{tabular}{|c|c|c|c|c|c|c|c|c|c|c|}
\hline $\begin{array}{l}\text { Specimen } \\
\text { Name }\end{array}$ & $\# 1$ & $\# 2$ & $\# 3$ & $\# 4$ & \#5 & $\# 6$ & $\# 7$ & $\# 8$ & $\# 9$ & $\# 10$ \\
\hline Porosity (\%) & 0.7 & 3.2 & 5.2 & 7.6 & 11 & 13 & 23 & 26 & 29 & 39 \\
\hline Porosity Level & $\begin{array}{c}\text { Lev. } 1 \\
(0-2.5)\end{array}$ & $\begin{array}{l}\text { Lev. } 2 \\
(2.5-5)\end{array}$ & $\begin{array}{l}\text { Lev. } 3 \\
(5-7.5)\end{array}$ & $\begin{array}{c}\text { Lev. } 4 \\
(7.5-10)\end{array}$ & $\begin{array}{c}\text { Lev. } 5 \\
(10-12.5)\end{array}$ & $\begin{array}{c}\text { Lev. } 6 \\
(12.5-15)\end{array}$ & $\begin{array}{c}\text { Lev. } 7 \\
(22.5-25)\end{array}$ & $\begin{array}{c}\text { Lev. } 8 \\
(25-27.5)\end{array}$ & $\begin{array}{c}\text { Lev. } 9 \\
(27.5-30)\end{array}$ & $\begin{array}{l}\text { Lev. } 10 \\
(30-)\end{array}$ \\
\hline
\end{tabular}

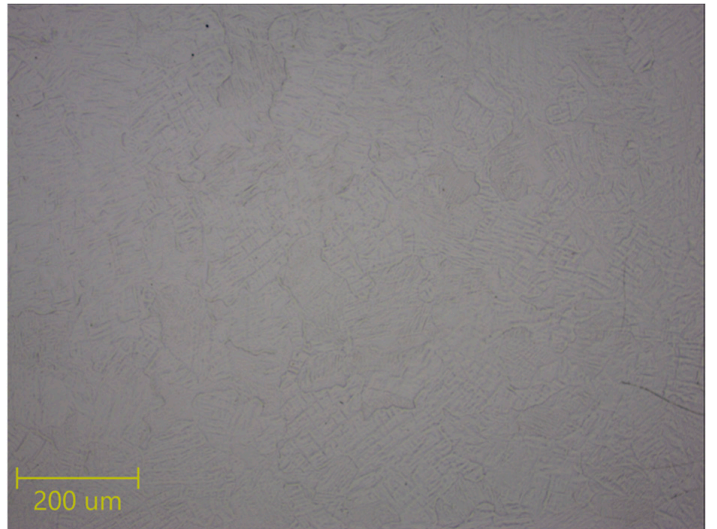

(a)

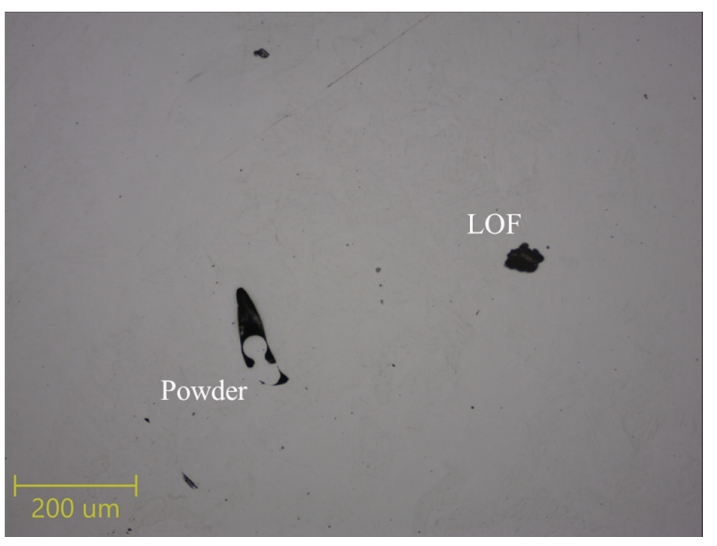

(c)

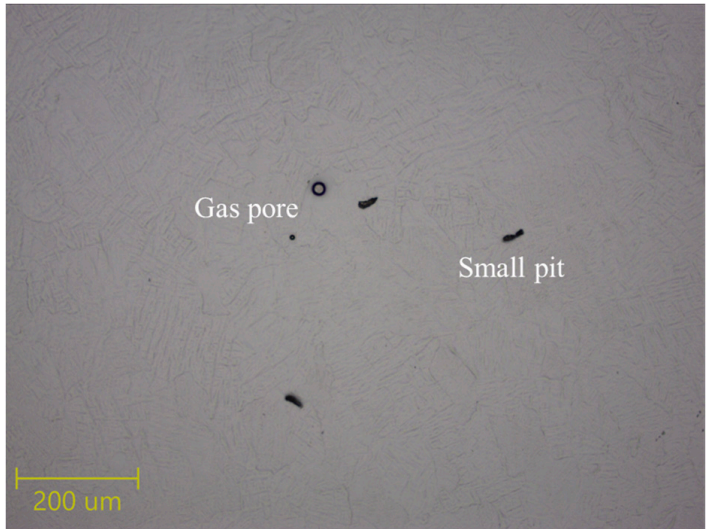

(b)

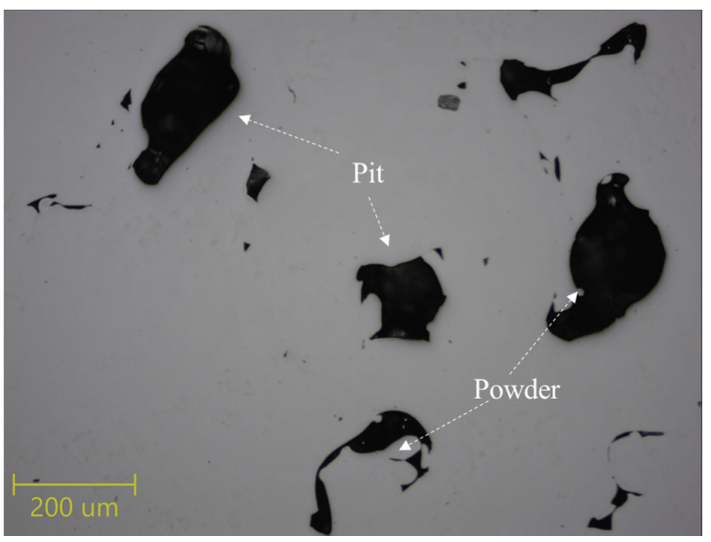

(d)

Figure 3. OM images of specimens (a)\#1, (b) \#3, (c) \#6, and (d) \#10. 


\subsection{Ultrasonic Measurements}

Ultrasonic measurements were conducted by a pulse-echo mode using a contact transducer. This method is a well-known nondestructive testing technique that uses a pulsed signal with a broad bandwidth and several back-wall echo signals [50]. A schematic diagram and an image of the experimental setup are shown in Figure 4. A pulsed voltage signal, generated by a commercial pulser/receiver, was sent to a $5 \mathrm{MHz}$ piezoelectric transducer. A longitudinal wave with a wavelength of approximately $1.2 \mathrm{~mm}$ was emitted by the transducer and was then incident on the AM specimen. At this wavelength, the ultrasonic diffraction effect is negligibly small because the ultrasonic wave propagation distance is in the range of the near field zone, which is obtained by $D^{2} / 4 \lambda=19 \mathrm{~mm}$, where $D$ is the transducer diameter and $\lambda$ is the wavelength. The back-wall echo was received by the same transducer, and the ultrasonic signal was displayed and saved on a commercial oscilloscope. This echo signal reflects the effects of porosity in the ultrasonic propagation direction in the form of variations in the ultrasonic arrival time and ultrasonic attenuations [50]. To minimize the ultrasonic measurement errors, a pneumatic device that can apply a consistent pressure of $0.4 \mathrm{MPa}$ was used, such that the contact condition between the transducer and the specimen was maintained consistently in each measurement [50]. Ultrasonic signals were obtained for the three different surface conditions: smooth, medium, and rough Typical signals obtained for specimens \#1 and \#1' prepared with three different surface conditions are plotted in Figure 5. The three measured signals overlap with each other. Although the porosity contents of specimens $\# 1$ and $\# 1^{\prime}$ are the same, the difference in their ultrasonic amplitudes is clearly visible when the three signals are compared. In particular, the amplitude loss is very large on the surface of the "rough condition" specimen. The SNRs of these signals were $33 \mathrm{~dB}, 16 \mathrm{~dB}$, and $10 \mathrm{~dB}$, respectively, as shown in Figure 5 . These SNRs are attributed to imperfect contact between the transducer and test specimens. The presence of air gaps owing to the imperfect contact results in impedance mismatch and also multi-reflections of the incident ultrasonic waves. Consequently, except for the "smooth condition", the additional changes in the properties of the incident ultrasonic waves make it difficult to evaluate the porosity of rough surfaces by using conventional UT such as ultrasonic velocity and ultrasonic attenuation coefficient measurements [22].

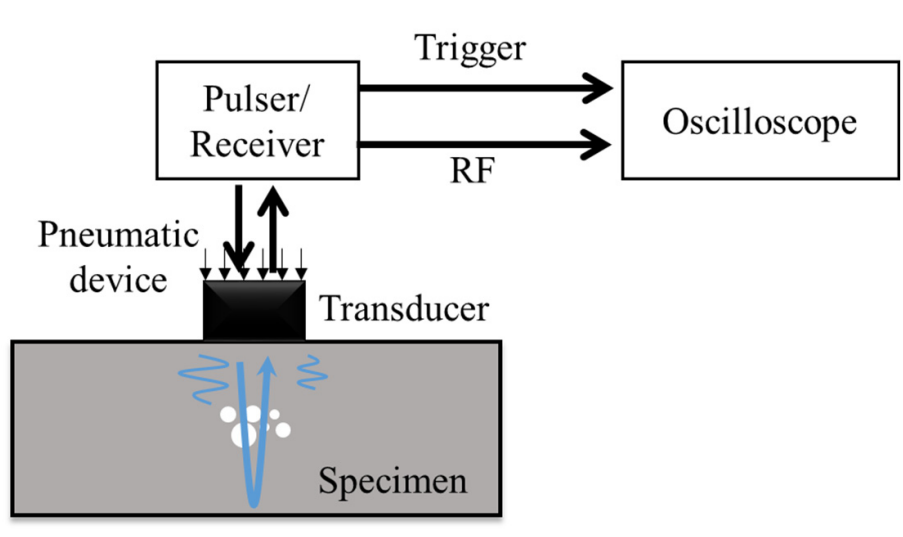

(a)

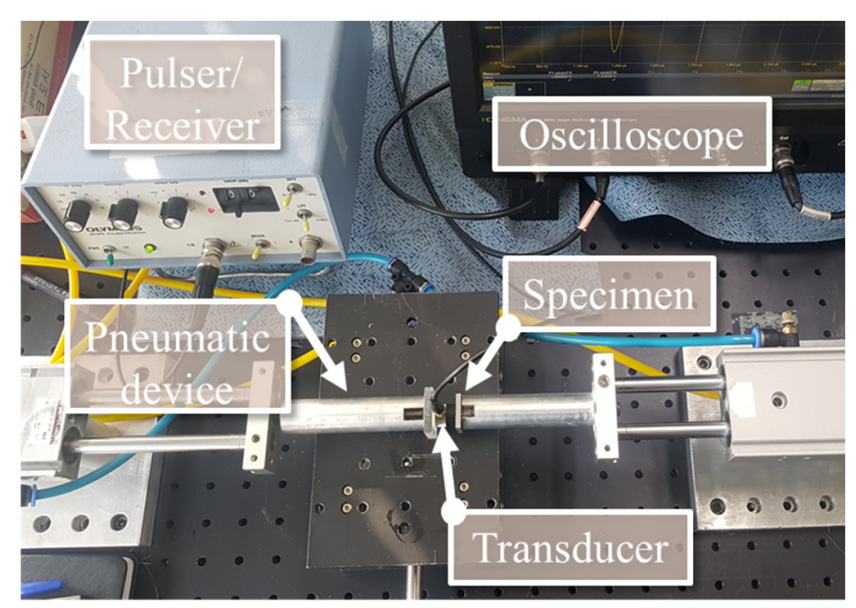

(b)

Figure 4. (a) Schematic diagram and (b) image of the experimental setup. 


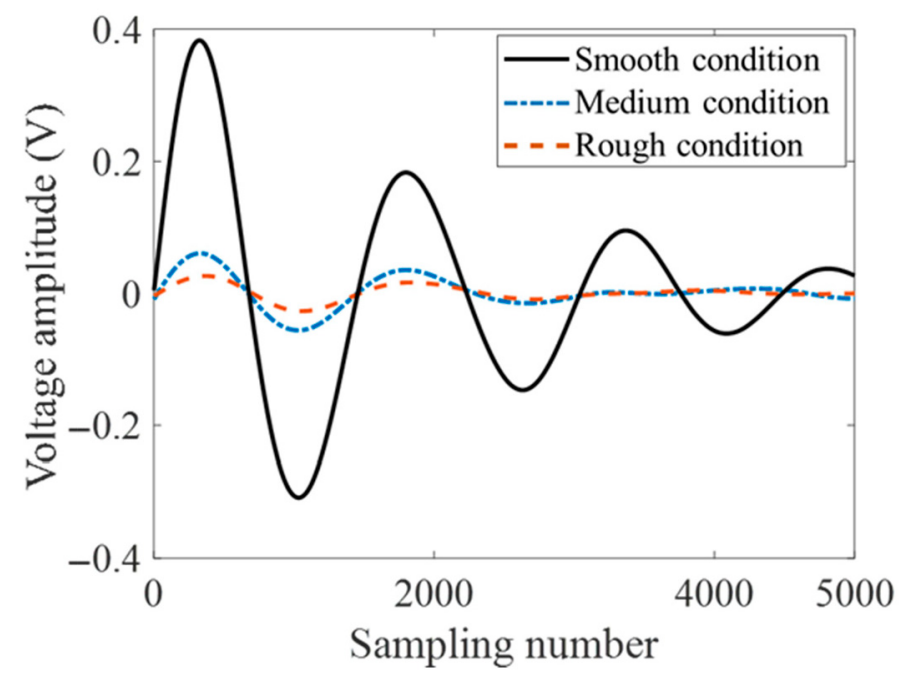

Figure 5. Ultrasonic signals obtained from the three different surface conditions. The signals were cropped to 5000 sampling numbers and were plotted to overlap with each other.

\section{Porosity Evaluation in Rough Surface Conditions}

\subsection{Structures of Deep Learning Models}

Artificial intelligence using neural network uses the group of correlated nodes motivated by biological neurons [51,52]. The simplest model is the MLP, which consists of an input layer, an output layer, and one hidden layer, where nodes are fully connected with each other [53]. DNN consists of more than two hidden layers with input and output layers, with the deeper structures being able to enhance the feature extraction capability. CNN is a type of MLP designed to use a feature extractor that requires minimal pre-processing with a fully connected neural network [51,54]. The feature extractor consists of one or more convolutional and pooling layers. A high-level feature map obtained from the feature extractor enables the network to be deeper with fewer parameters [55].

In this study, the CNN, DNN, and MLP models were used, and their performance was compared. Among several types of CNN, a one-dimensional (1D) CNN, which is effective not only to derive features from shorter segments of overall data but also accepts any type of signal as the input, was used. This network comprised a 1D array-type input layer, two convolutional layers, two max-pooling layers, a fully connected layer, and an output layer [56]. The input layer was restricted to $(5000 \times 1)$ nodes, which corresponded to the sampling numbers of the original ultrasonic signals. Note that the down-sampling of input nodes from original signals can reduce the computation time during model training. However, this down-sampling can also decrease the ability of the DL model to extract porosity features in the ultrasonic signals. In the first convolutional layer, the kernel size was set wide with $(50 \times 1)$ to restrain noise effectively, and the sizes of feature map and stride were 32 and $(5 \times 1)$, respectively. In the second convolutional layer, the kernel size was set to $(4 \times 1)$, considerably smaller than the first layer to extract a large number of features. The sizes of the feature map and stride in the second layer were 64 and $(2 \times 1)$, respectively. According to several simulation studies [34,35], these two convolutional layers with different kernel sizes showed good performance in noisy conditions. After each convolutional layer, one pooling layer was used, where both pooling and stride sizes were $(2 \times 1)$. The fully connected layer was set to $(1000 \times 1)$ nodes and connected to the output layer based on the softmax function $F\left(s_{i}\right)$ with cross-entropy $(C E)$ loss for classification, derived as follows [34]:

$$
\begin{gathered}
F\left(s_{i}\right)=\frac{e^{s_{i}}}{\sum_{j=1}^{K} e^{s_{j}},} \\
C E=-\sum_{i}^{K} t_{i} \log \left(s_{i}\right),
\end{gathered}
$$


where $s$ is the predicted output, subscript $i$ indexes each output class, $K$ is the total class numbers, and $t_{i}$ is the real output. The activation function was a rectified linear unit (ReLU), presented as follows [57]:

$$
\operatorname{ReLU}(z)=\max (0, z) .
$$

To prevent an overfitting problem, dropout regularization [58] with a $70 \%$ training probability, which is a trick method to deactivate several nodes during training, was used before and after the fully connected layer. This dropout is also effective for the stronger robustness of the model. The learning rate was set to 0.001 after several trials. Note that too large a learning rate shows a corresponding effect for the down-sampling of the input nodes. Details of the CNN model are shown in Figure 6a. In the fully connected DNN model, instead of using the convolutional layer, the fully connected layers were set deeper than the used CNN model. Two hidden layers, i.e., $(1000 \times 1)$ and $(1000 \times 1)$ nodes, were used. The previous simulation studies $[34,35]$ also reported that the deeper structures showed a better feature extraction ability of the DNN model. In the MLP model, only one hidden layer with $(1000 \times 1)$ nodes was used. The other parameters of the DNN and MLP models, such as the number of nodes of the input and output, and the dropout rate, were set to correspond to those of the CNN model, as shown in Figure $6 \mathrm{~b}, \mathrm{c}$, and Table 4 . The Relu and $F\left(s_{i}\right)$ with CE functions were also used. All the models were designed using TensorFlow and Keras.

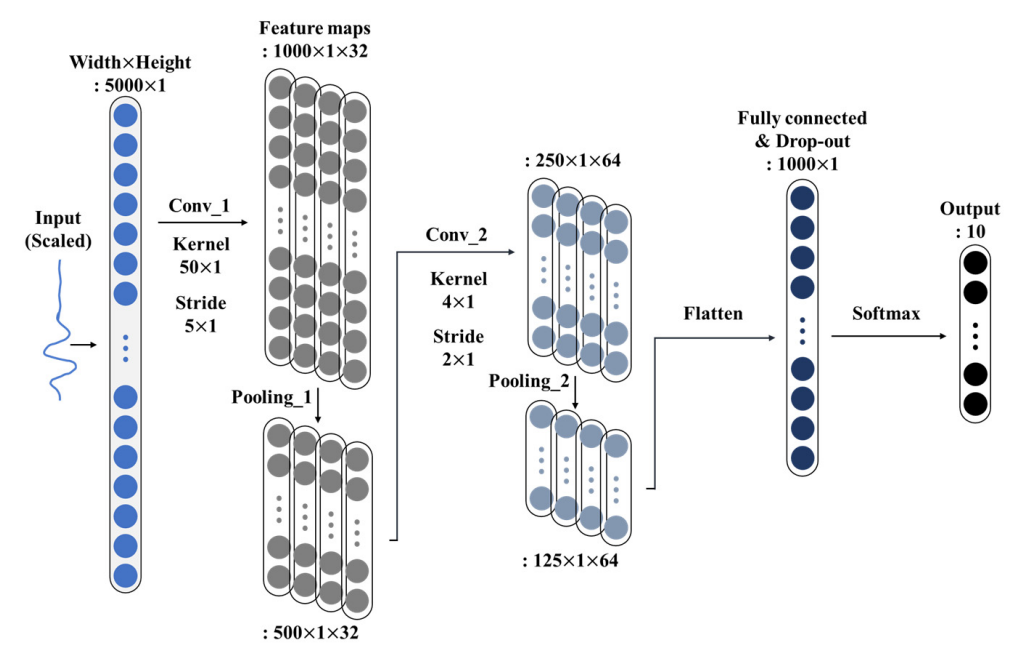

(a)

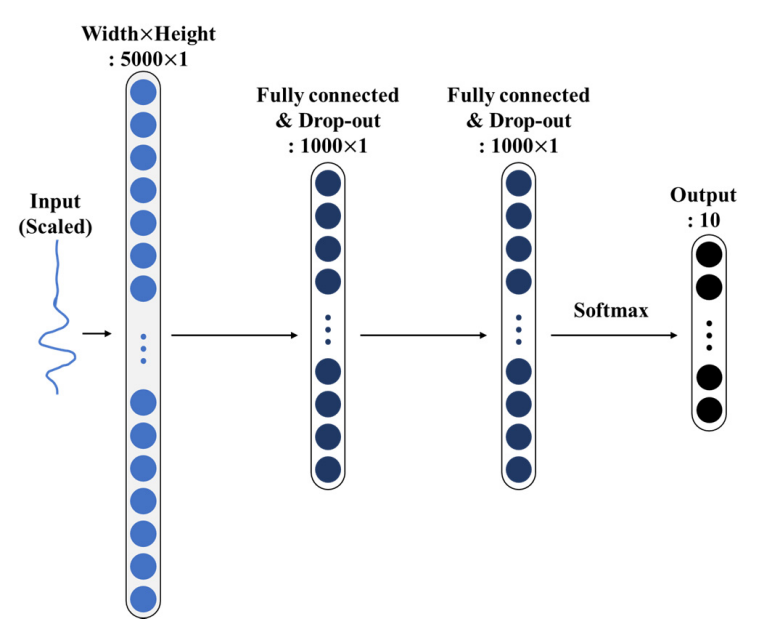

(b)

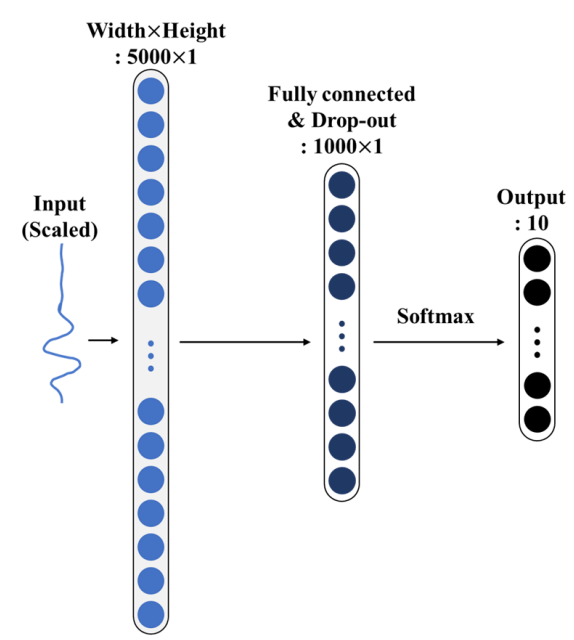

(c)

Figure 6. Architectures of the (a) convolutional neural network (CNN), (b) deep neural network (DNN), and (c) multi-layer perceptron (MLP) models. 
Table 4. Parameters used to train each model.

\begin{tabular}{cccc}
\hline Model & CNN & DNN & MLP \\
\hline Input & 5000 & 5000 & 5000 \\
1st conv: Feature map/Kernel/Stride & $32 / 50 / 5$ & - & - \\
1st max-pooling: Kernel/Stride & $2 / 2$ & - & - \\
2nd conv: Feature map/Kernel/Stride & $64 / 4 / 2$ & - & - \\
2nd max-pooling: Kernel/Stride & $2 / 2$ & - & - \\
Fully connected (Wide) & 1000 & 1000 & 1000 \\
Fully connected (Deep) & 1 & 2 & 1 \\
Drop out & $70 \%$ & 0.001 & $70 \%$ \\
Learning rate & 0.001 & Softmax cross-entropy & Softmax cross-entropy \\
Cost function & Softmax cross-entropy & ReLU & ReLU \\
Activation function & ReLU &
\end{tabular}

\subsection{Procedures to Train and Test the Models}

The CNN, DNN, and MLP models were trained using the prepared training dataset to derive each specific function for porosity evaluation, after which the testing performance was compared. Training was conducted by using a classification method based on supervised learning for three models, with the respective training datasets generating the class label as the output. Ten labeled classes with porosity levels ranging from 1 to 10 were used with the levels based on the results of the porosity content measurements obtained with SAM. Ultrasonic signals were acquired from the surfaces with three different roughness levels. For each surface roughness level, 100 ultrasonic signals were measured, of which 80 ultrasonic signals were randomly extracted and used as the training dataset. The remaining 20 signals were used as the testing dataset. Considering previous research [36], the amount of data used to form the training and testing datasets is sufficient. The training and testing datasets for each of the 10 porosity levels and the three different roughness conditions are summarized in Table 5.

Table 5. Training and testing datasets according to roughness condition and porosity level.

\begin{tabular}{|c|c|c|c|c|c|c|c|c|c|}
\hline \multirow{3}{*}{$\begin{array}{c}\text { Porosity } \\
\text { Level }\end{array}$} & \multicolumn{3}{|c|}{ Smooth Condition } & \multicolumn{3}{|c|}{ Medium Condition } & \multicolumn{3}{|c|}{ Rough Condition } \\
\hline & \multirow{2}{*}{ Specimen } & \multicolumn{2}{|c|}{ No. of Signals } & \multirow{2}{*}{ Specimen } & \multicolumn{2}{|c|}{ No. of Signals } & \multirow{2}{*}{ Specimen } & \multicolumn{2}{|c|}{ No. of Signals } \\
\hline & & Train & Test & & Train & Test & & Train & Test \\
\hline Lev. 1 & $\# 1$ & 80 & 20 & $\# 1^{\prime}$ & 80 & 20 & $\# 1^{\prime}$ & 80 & 20 \\
\hline Lev. 2 & \#2 & 80 & 20 & $\# 2^{\prime}$ & 80 & 20 & $\# 2^{\prime}$ & 80 & 20 \\
\hline Lev. 3 & \#3 & 80 & 20 & $\# 3^{\prime}$ & 80 & 20 & $\# 3^{\prime}$ & 80 & 20 \\
\hline Lev. 4 & $\# 4$ & 80 & 20 & $\# 4^{\prime}$ & 80 & 20 & $\# 4^{\prime}$ & 80 & 20 \\
\hline Lev. 5 & $\# 5$ & 80 & 20 & $\# 5^{\prime}$ & 80 & 20 & $\# 5^{\prime}$ & 80 & 20 \\
\hline Lev. 6 & \#6 & 80 & 20 & $\# 6^{\prime}$ & 80 & 20 & $\# 6^{\prime}$ & 80 & 20 \\
\hline Lev. 7 & $\# 7$ & 80 & 20 & $\# 7^{\prime}$ & 80 & 20 & $\# 7^{\prime}$ & 80 & 20 \\
\hline Lev. 8 & $\# 8$ & 80 & 20 & $\# 8^{\prime}$ & 80 & 20 & $\# 8^{\prime}$ & 80 & 20 \\
\hline Lev. 9 & $\# 9$ & 80 & 20 & $\# 9^{\prime}$ & 80 & 20 & $\# 9^{\prime}$ & 80 & 20 \\
\hline Lev. 10 & $\# 10$ & 80 & 20 & $\# 10^{\prime}$ & 80 & 20 & $\# 10^{\prime}$ & 80 & 20 \\
\hline
\end{tabular}

Figure 7 shows learning curves of the CNN, DNN, and MLP models for the "rough condition", which represents the testing accuracy and cost as a function of the number of epochs. The testing accuracy was defined as the classification performance at each epoch on the testing data, and was calculated as:

$$
\text { Testing accuracy }(\%)=m_{1} / n_{1} \cdot 100 \text {, }
$$

where $m_{1}$ is the number of testing data points classified well, and $n_{1}$ is the overall number of testing data points. The performance of the model can be evaluated from the testing accuracy, which represents the classification performance at each epoch on the testing 
data. The cost represents the error between the real output and the predicted output of the tested model based on the testing dataset. Figure 7 shows that the testing accuracy of the respective CNN, DNN, and MLP model reached a global maximum and oscillated after approximately the 40th epoch. Therefore, we monitored the testing accuracy from the 40th epoch until the end, and the epoch that provided the highest testing accuracy was chosen for the respective CNN, DNN, and MLP model [59]. A commercial CPU device was used to train all models. The computation time for the respective CNN, DNN, and MLP model was approximately 760,500 , and $96 \mathrm{~s}$, respectively.

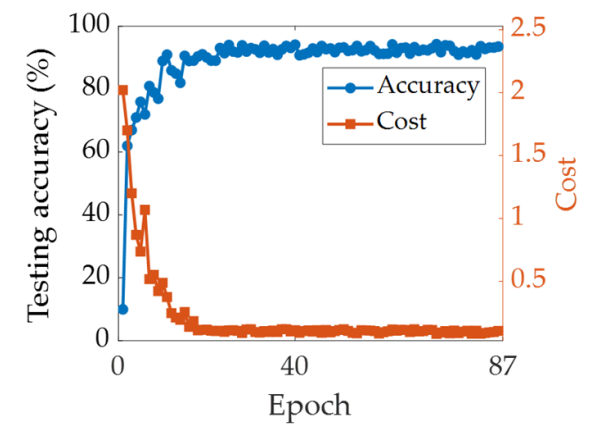

(a)

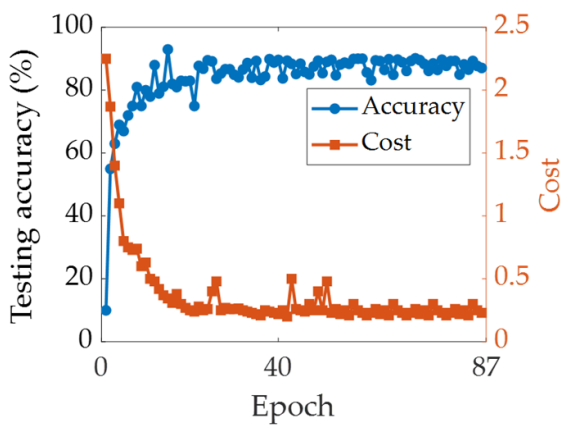

(b)

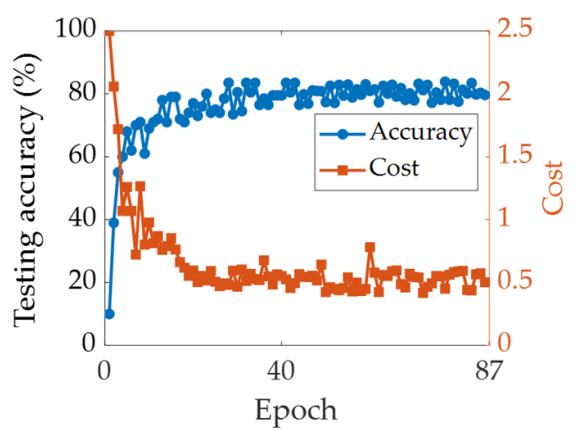

(c)

Figure 7. Learning curves of the (a) CNN, (b) DNN, and (c) MLP models for the "rough condition", showing the testing accuracy and cost with respect to the number of epochs.

Table 6 lists the testing performance of the three different models for the various surface roughness levels, and these results are compared in Figure 8. The testing performance results are also listed as confusion matrices in Appendix A. For the "smooth condition" surface, each CNN and DNN model delivered average testing performance of $98.5 \%$ and $98.0 \%$, respectively. Although the performance of the MLP model was relatively lower than that of the others, it also performed well at $96.0 \%$. Increased roughness levels caused the performance of all the models to decrease; however, the performance of all the models exceeded $80.5 \%$ in terms of their accuracy. The model that delivered the best performance for the "medium condition" and "rough condition" surfaces was CNN. The performance only decreased to $97.5 \%$ and $94.5 \%$ for surfaces with these two roughness levels, i.e., decreases of $1 \%$ and $4 \%$, respectively, when compared with the "smooth condition" surface. Those of the DNN were $95.5 \%$ and $89.5 \%$ for the "medium condition" and "rough condition", respectively, a slightly larger decrease of $2.5 \%$ and $8.5 \%$, respectively. This tendency is more pronounced for the MLP model, in which case the performance decreased to $92.0 \%$ and $80.5 \%$, i.e., decreases of $4 \%$ and $16.5 \%$, respectively. In comparison, the performance of all models decreased slightly at porosity levels 1 and 2 compared with the other levels regardless of the surface roughness conditions. Above porosity level 3 , the average performance for all roughness conditions for each of the CNN, DNN, and MLP models was $99.4 \%, 96.3 \%$, and $92.5 \%$, respectively. However, below level 2, these values decreased to $86.7 \%, 86.7 \%$, and $77.5 \%$, respectively.

Conventional UT is based on ultrasonic velocity and ultrasonic attenuation coefficient measurements [13]. The use of these methods requires not only the first back-wall echo signal but also the second echo in pulse-echo mode to be measured to extract the ultrasonic velocity and attenuation coefficient parameters. A comparison of the extent to which the parameters vary enables the porosity to be evaluated. These parameters are calculated as follows [13]:

$$
\begin{gathered}
v=\frac{d}{\tau}, \\
a=\frac{20}{d} \log \left(\frac{A_{1}}{A_{2}}\right),
\end{gathered}
$$


where $v$ is the ultrasonic velocity, $d$ is the ultrasonic wave propagation distance corresponding to twice the thickness of the specimen, $\tau$ is the time-of-flight difference between two consecutive echoes, $a$ is the ultrasonic attenuation coefficient, and $A_{1}$ and $A_{2}$ are the amplitudes of two consecutive echoes, respectively. Generally, the amplitude of the second echo is smaller than that of the first echo because the second echo is propagated over a longer distance.

Table 6. Testing performance of the three different models for the three different surface roughness conditions.

\begin{tabular}{cccccccccc}
\hline & \multicolumn{8}{c}{ Testing Performance (\%) } \\
\cline { 2 - 9 } Classification & \multicolumn{2}{c}{ Smooth Condition } & \multicolumn{1}{c}{ Medium Condition } & \multicolumn{2}{c}{ Rough Condition } \\
\cline { 2 - 10 } & CNN & DNN & MLP & CNN & DNN & MLP & CNN & DNN & MLP \\
\hline Lev. 1 & 90 & 95 & 85 & 85 & 90 & 80 & 75 & 70 & 60 \\
Lev. 2 & 95 & 95 & 90 & 90 & 90 & 80 & 85 & 80 & 70 \\
Lev. 3 & 100 & 100 & 95 & 100 & 95 & 90 & 90 & 90 & 85 \\
Lev. 4 & 100 & 100 & 95 & 100 & 95 & 95 & 100 & 85 & 75 \\
Lev. 5 & 100 & 95 & 100 & 100 & 95 & 95 & 95 & 90 & 85 \\
Lev. 6 & 100 & 100 & 100 & 100 & 100 & 100 & 100 & 95 & 85 \\
Lev. 7 & 100 & 100 & 100 & 100 & 100 & 95 & 100 & 100 & 95 \\
Lev. 8 & 100 & 95 & 100 & 100 & 95 & 95 & 100 & 95 & 90 \\
Lev. 9 & 100 & 100 & 100 & 100 & 95 & 100 & 100 & 90 & 80 \\
Lev. 10 & 100 & 100 & 95 & 100 & 100 & 90 & 100 & 100 & 80 \\
\hline Average & $\mathbf{9 8 . 5}$ & $\mathbf{9 8}$ & $\mathbf{9 6}$ & $\mathbf{9 7 . 5}$ & $\mathbf{9 5 . 5}$ & $\mathbf{9 2}$ & $\mathbf{9 4 . 5}$ & $\mathbf{8 9 . 5}$ & $\mathbf{8 0 . 5}$ \\
\hline
\end{tabular}

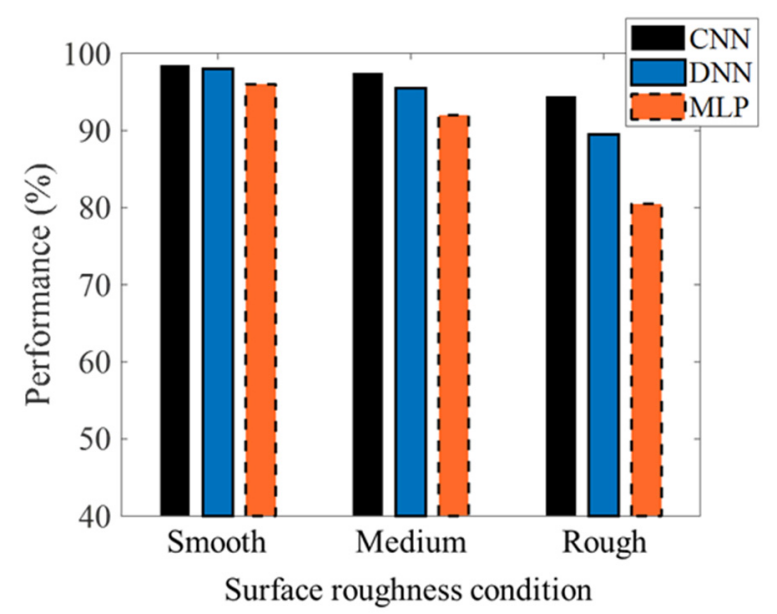

Figure 8. Performance of the CNN, DNN, and MLP models for the three different surface roughness conditions.

Figure 9 shows two consecutive echoes measured from specimens with the three surface roughness levels. For the "smooth condition" two echoes are clearly observed. However, for the other roughness levels, the levels of the second echo and background noise are almost similar owing to the amplitude loss from the rough surfaces. Consequently, rough surface conditions make it difficult to employ conventional UT for porosity evaluation.

Several reasons could exist for the high performance of the DL models in terms of their porosity evaluation of AM parts with rough surfaces. The first simple reason is their excellent ability to perform feature extraction. The use of DL models with deep and wide structures with hidden nodes is known to be more effective for extracting features than conventional UT [36]. The second reason is that the training dataset of the DL model consists of the raw ultrasonic signals, whereas conventional UT, which includes the 
ultrasonic velocity and attenuation measurements, only uses the velocity and attenuation coefficient parameters extracted from the ultrasonic signals. When the raw signal is used for training, various properties including not only the velocity and attenuation but also the ultrasonic backscattering and non-linearity can be used as features. Although not to the same extent as the velocity and attenuation, backscattering and non-linearity are also known to be related to the porosity content, which enhances the performance when DL models are used [50]. Our experimental results showed that the rougher the surface, that is, the lower the SNR, the more effective is the DL model. At the same time, the CNN model outperformed the DNN and MLP models because the CNN model, which uses a pre-processor, is beneficial for feature extraction from the waveform even for low SNRs. The waveform of the ultrasonic wave propagating through the porous medium varied locally. As mentioned above, the typical waveform variation is the delay in the arrival time and ultrasonic attenuation owing to local elastic inhomogeneity at the boundary of the pores. When the CNN model is used, both the convolutional and pooling layers in the pre-processor assign a greater weight to this variation in the waveform, thereby enabling the $\mathrm{CNN}$ to achieve more effective feature extraction than the other models.

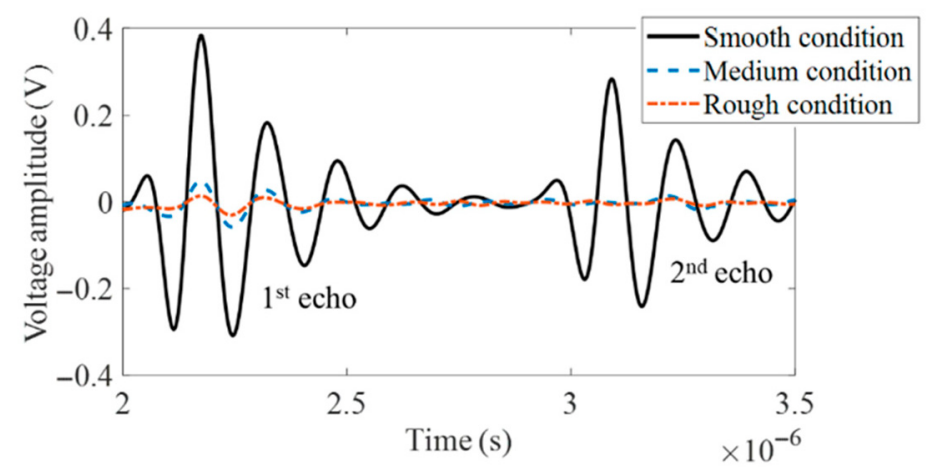

Figure 9. Ultrasonic 1st and 2nd echo signals measured from surfaces with the "smooth condition", "medium condition", and "rough condition".

Note that, in addition to the surface roughness issue, porosity with an irregular distribution pattern may affect the UT performance. For example, if the porosity is distributed non-uniformly in the direction parallel to the surface attached to the ultrasonic transducer, the UT performance may deteriorate depending on the positions at which measurements are conducted (where the surface in contact with the transducer is assumed to be constant). Generally, porosity originates from a lack of uniformity along the building direction because the cooling rate is varied during AM building. In contrast, the plane normal to the building direction is relatively uniform [21]. In our experiments, the ultrasonic measurement was conducted using a transducer attached to the surface in the direction normal to the building direction, as shown in Figure 4. In other words, an ultrasonic wave propagating in a direction parallel to the building direction reflects the effects of a non-uniform pore; however, the average porosity along this path is almost uniform in the direction parallel to the surface attached to the transducer. Therefore, there may be few errors in the UT performance owing to the irregular pattern in which the porosity is distributed. However, in the case of low levels of porosity, this assumption may be difficult to establish. In fact, our experimental results indicated that, below porosity level 2, the performance is slightly lower.

\subsection{Evaluation of the Generalization Performance}

To verify the applicability of the pre-trained model, a generalization performance test was carried out on newly fabricated AM specimens, which were not utilized to train the models. The generalization test was conducted on specimens in the as-deposited condition, i.e., the "rough condition". Only the pre-trained CNN model, which delivered the best performance for this roughness condition, was used. Two new specimens were manufactured 
by using the same AM process but different AM processing parameters. These parameters did not correspond to the processing conditions of the existing $10 \mathrm{AM}$ specimens that were used to train the models. One-hundred ultrasonic signals were obtained for each specimen and were used as input to the pre-trained model. Figure 10 shows the results of the generalization performance of the two AM specimens using the pre-trained CNN model. This model assessed the Test\#1 specimen as "Porosity level 2" with the highest probability of $89 \%$ and "Porosity level 1" with the second highest of $8 \%$. This model also rated the Test-\#2 specimen as "Porosity level 8" with 91\% and "Porosity level 7" with 7\%.

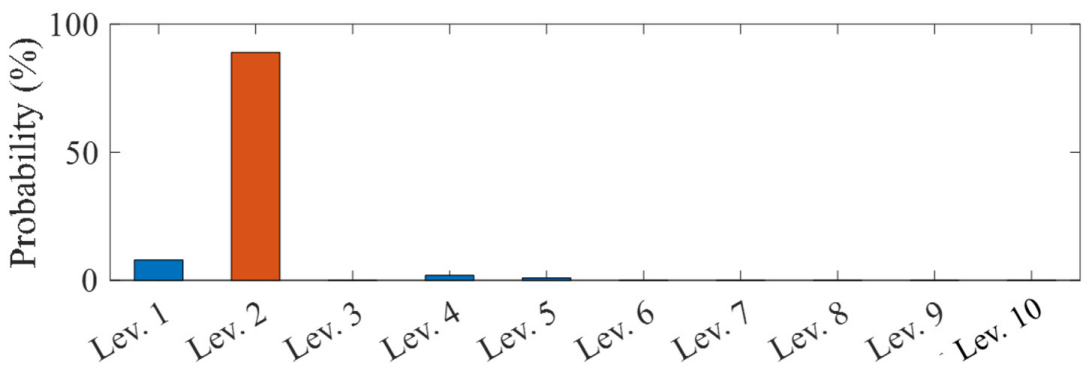

(a)

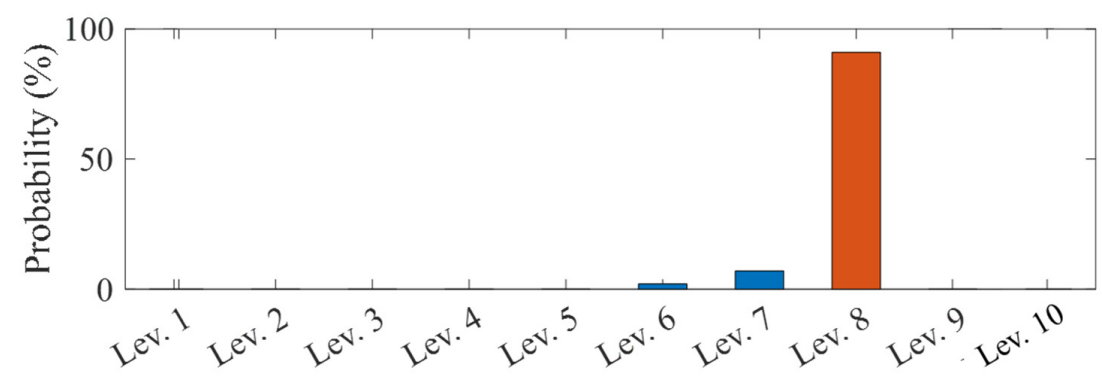

(b)

Figure 10. Generalization test results for the (a) Test-\#1 and (b) Test-\#2 specimens.

To validate the results obtained by the pre-trained CNN model, SAM was also used to assess the porosity content of the tested specimens. Because SAM cannot be employed to examine as-deposited specimens with rough surfaces, the test specimens were additionally polished using wire EDM. Figure 11 shows the obtained C-mode images. The porosity contents that were calculated from these images are presented in Table 7 alongside the assessment with the pre-trained CNN model. The calculated porosity contents of Test\#1 and Test\#2 were $4.3 \%$ and $27 \%$, respectively, which were within the range of "Porosity level 2" and "Porosity level 8", respectively. In other words, the SAM results were in good correspondence with the results assessed as having the highest probability by the CNN model. In addition, the average generalization performance for the "rough condition" was $90 \%$, which is slightly lower than the testing performance in Section 3.2. This might be due to differences in the AM processing conditions [1] and the experimental environment.

Table 7. Comparison of the results obtained with the pre-trained model and SAM.

\begin{tabular}{cccc}
\hline \multirow{2}{*}{ AM Specimen } & \multicolumn{3}{c}{ Porosity Evaluation Results } \\
\cline { 2 - 4 } & \multicolumn{2}{c}{ Pre-Trained Model } & SAM \\
\cline { 2 - 4 } & Porosity Level & Porosity Content (\%) & Porosity Content (\%) \\
\hline Test-\#1 & Lev. 2 & $2.5-5$ & 4.3 \\
Test-\#2 & Lev. 8 & $25-27.5$ & 27 \\
\hline
\end{tabular}




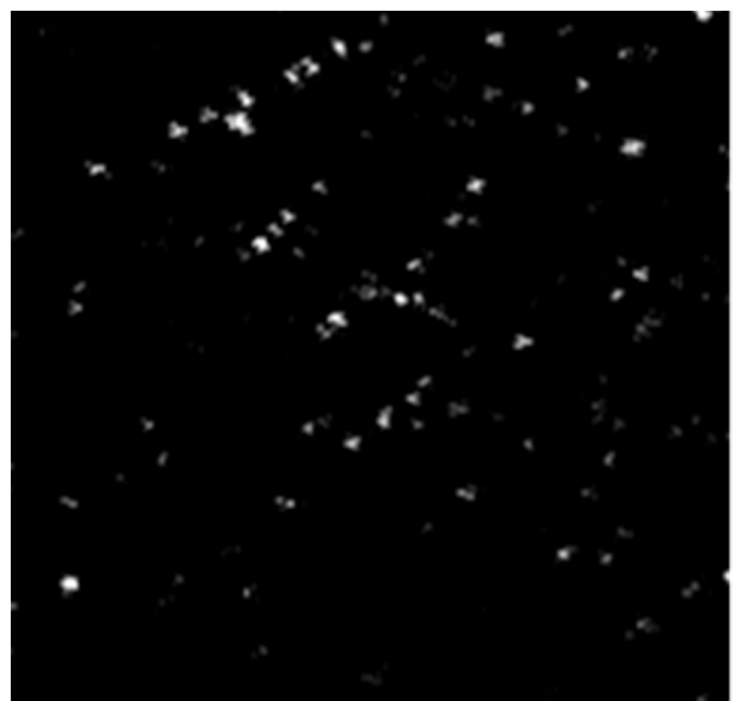

(a)

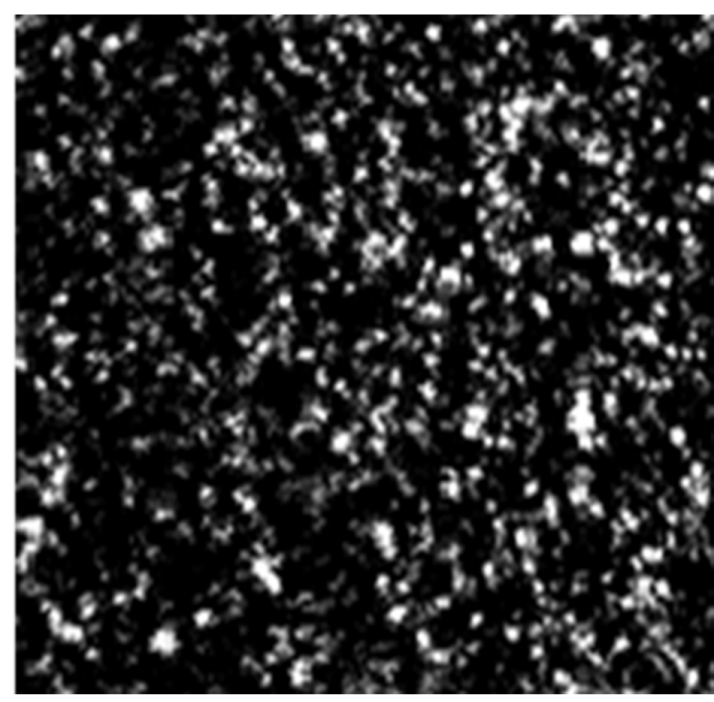

(b)

Figure 11. SAM results for the (a) Test-\#1 and (b) Test-\#2 specimens.

\section{Conclusions}

In this work, DL techniques were used in conjunction with UT to evaluate the porosity of AM parts with rough surfaces. Key research outcomes were as follows.

(1) Various porosity mechanisms were investigated through SAM and OM analysis. Porosity contents increased in the order of normal (the relative porosity content measured by SAM: $0.7 \%)$, over-melting $(4.2 \%)$, LOF (16.1\%), and over-melting with low laser power conditions (34\%).

(2) A comparison of the performance results of the various DL models showed that all the models were highly accurate at over $80.5 \%$, even for the as-deposited specimens with surfaces in the "rough condition". In particular, CNN was the most effective at $94.5 \%$. Owing to the low SNR of the measured ultrasonic signal, conventional UT using ultrasonic velocity and ultrasonic attenuation coefficient measurements could not be used to assess "medium condition" and "rough condition" surfaces.

(3) A generalization test was also conducted using newly as-deposited AM specimens that were not used for training to evaluate the applicability of the pre-trained CNN model. The test results confirmed the model's high evaluation performance of $90.0 \%$, which corresponded well with the results obtained with SAM.

These results suggest that the use of DL could be expected to enhance the UT performance with respect to the porosity evaluation of AM parts, even for as-deposited rough surfaces.

Author Contributions: Conceptualization, S.-H.P.; methodology, S.-H.P., S.C. and K.-Y.J.; validation, S.-H.P., J.-Y.H., T.H. and S.C.; formal analysis, S.-H.P., J.-Y.H., T.H. and S.C.; investigation, S.-H.P. and J.-Y.H.; resources, S.-H.P., J.-Y.H. and K.-Y.J.; writing—original draft preparation, S.-H.P.; writingreview and editing, S.-H.P., S.C. and K.-Y.J.; supervision, S.C. and K.-Y.J.; funding acquisition, T.H. and K.-Y.J. All authors have read and agreed to the published version of the manuscript.

Funding: This work was supported by a Korea Institute of Machinery and Materials grant funded by the Korea government (MSIT) (NK230l), and the Korea Institute of Energy Technology Evaluation and Planning (KETEP) and the Ministry of Trade, Industry and Energy (MOTIE) of the Republic of Korea (No.20181510102360).

Institutional Review Board Statement: Not applicable.

Informed Consent Statement: Not applicable.

Conflicts of Interest: The authors declare no conflict of interest. 


\section{Appendix A}

Table A1. Confusion matrices for the CNN model in three surface conditions.

\begin{tabular}{|c|c|c|c|c|c|c|c|c|c|c|}
\hline \multicolumn{11}{|c|}{ CNN } \\
\hline \multicolumn{11}{|c|}{ Smooth condition } \\
\hline (Unit: \%) & Lev. 1 & Lev. 2 & Lev. 3 & Lev. 4 & Lev. 5 & Lev. 6 & Lev. 7 & Lev. 8 & Lev. 9 & Lev. 10 \\
\hline Lev. 1 & 90 & 10 & 0 & 0 & 0 & 0 & 0 & 0 & 0 & 0 \\
\hline Lev. 2 & 5 & 95 & 0 & 0 & 0 & 0 & 0 & 0 & 0 & 0 \\
\hline Lev. 3 & 0 & 0 & 100 & 0 & 0 & 0 & 0 & 0 & 0 & 0 \\
\hline Lev. 4 & 0 & 0 & 0 & 100 & 0 & 0 & 0 & 0 & 0 & 0 \\
\hline Lev. 5 & 0 & 0 & 0 & 0 & 100 & 0 & 0 & 0 & 0 & 0 \\
\hline Lev. 6 & 0 & 0 & 0 & 0 & 0 & 100 & 0 & 0 & 0 & 0 \\
\hline Lev. 7 & 0 & 0 & 0 & 0 & 0 & 0 & 100 & 0 & 0 & 0 \\
\hline Lev. 8 & 0 & 0 & 0 & 0 & 0 & 0 & 0 & 100 & 0 & 0 \\
\hline Lev. 9 & 0 & 0 & 0 & 0 & 0 & 0 & 0 & 0 & 100 & 0 \\
\hline Lev. 10 & 0 & 0 & 0 & 0 & 0 & 0 & 0 & 0 & 0 & 100 \\
\hline \multicolumn{11}{|c|}{ Medium condition } \\
\hline (Unit: \%) & Lev. 1 & Lev. 2 & Lev. 3 & Lev. 4 & Lev. 5 & Lev. 6 & Lev. 7 & Lev. 8 & Lev. 9 & Lev. 10 \\
\hline Lev. 1 & 85 & 10 & 0 & 5 & 0 & 0 & 0 & 0 & 0 & 0 \\
\hline Lev. 2 & 10 & 90 & 0 & 0 & 0 & 0 & 0 & 0 & 0 & 0 \\
\hline Lev. 3 & 0 & 0 & 100 & 0 & 0 & 0 & 0 & 0 & 0 & 0 \\
\hline Lev. 4 & 0 & 0 & 0 & 100 & 0 & 0 & 0 & 0 & 0 & 0 \\
\hline Lev. 5 & 0 & 0 & 0 & 0 & 100 & 0 & 0 & 0 & 0 & 0 \\
\hline Lev. 6 & 0 & 0 & 0 & 0 & 0 & 100 & 0 & 0 & 0 & 0 \\
\hline Lev. 7 & 0 & 0 & 0 & 0 & 0 & 0 & 100 & 0 & 0 & 0 \\
\hline Lev. 8 & 0 & 0 & 0 & 0 & 0 & 0 & 0 & 100 & 0 & 0 \\
\hline Lev. 9 & 0 & 0 & 0 & 0 & 0 & 0 & 0 & 0 & 100 & 0 \\
\hline Lev. 10 & 0 & 0 & 0 & 0 & 0 & 0 & 0 & 0 & 0 & 100 \\
\hline \multicolumn{11}{|c|}{ Rough condition } \\
\hline (Unit: \%) & Lev. 1 & Lev. 2 & Lev. 3 & Lev. 4 & Lev. 5 & Lev. 6 & Lev. 7 & Lev. 8 & Lev. 9 & Lev. 10 \\
\hline Lev. 1 & 75 & 20 & 0 & 5 & 0 & 0 & 0 & 0 & 0 & 0 \\
\hline Lev. 2 & 10 & 85 & 5 & 0 & 0 & 0 & 0 & 0 & 0 & 0 \\
\hline Lev. 3 & 5 & 0 & 90 & 5 & 0 & 0 & 0 & 0 & 0 & 0 \\
\hline Lev. 4 & 0 & 0 & 0 & 100 & 0 & 0 & 0 & 0 & 0 & 0 \\
\hline Lev. 5 & 0 & 0 & 0 & 5 & 95 & 0 & 0 & 0 & 0 & 0 \\
\hline Lev. 6 & 0 & 0 & 0 & 0 & 0 & 100 & 0 & 0 & 0 & 0 \\
\hline Lev. 7 & 0 & 0 & 0 & 0 & 0 & 0 & 100 & 0 & 0 & 0 \\
\hline Lev. 8 & 0 & 0 & 0 & 0 & 0 & 0 & 0 & 100 & 0 & 0 \\
\hline Lev. 9 & 0 & 0 & 0 & 0 & 0 & 0 & 0 & 0 & 100 & 0 \\
\hline Lev. 10 & 0 & 0 & 0 & 0 & 0 & 0 & 0 & 0 & 0 & 100 \\
\hline
\end{tabular}

Table A2. Confusion matrices for the DNN model in three surface conditions.

\begin{tabular}{|c|c|c|c|c|c|c|c|c|c|c|}
\hline \multicolumn{11}{|c|}{ DNN } \\
\hline \multicolumn{11}{|c|}{ Smooth condition } \\
\hline (Unit: \%) & Lev. 1 & Lev. 2 & Lev. 3 & Lev. 4 & Lev. 5 & Lev. 6 & Lev. 7 & Lev. 8 & Lev. 9 & Lev. 10 \\
\hline Lev. 1 & 95 & 5 & 0 & 0 & 0 & 0 & 0 & 0 & 0 & 0 \\
\hline Lev. 2 & 5 & 95 & 0 & 0 & 0 & 0 & 0 & 0 & 0 & 0 \\
\hline Lev. 3 & 0 & 0 & 100 & 0 & 0 & 0 & 0 & 0 & 0 & 0 \\
\hline Lev. 4 & 0 & 0 & 0 & 100 & 0 & 0 & 0 & 0 & 0 & 0 \\
\hline Lev. 5 & 0 & 0 & 0 & 5 & 95 & 0 & 0 & 0 & 0 & 0 \\
\hline Lev. 6 & 0 & 0 & 0 & 0 & 0 & 100 & 0 & 0 & 0 & 0 \\
\hline Lev. 7 & 0 & 0 & 0 & 0 & 0 & 0 & 100 & 0 & 0 & 0 \\
\hline Lev. 8 & 0 & 0 & 0 & 0 & 0 & 0 & 0 & 95 & 5 & 0 \\
\hline Lev. 9 & 0 & 0 & 0 & 0 & 0 & 0 & 0 & 0 & 100 & 0 \\
\hline Lev. 10 & 0 & 0 & 0 & 0 & 0 & 0 & 0 & 0 & 0 & 100 \\
\hline
\end{tabular}


Table A2. Cont.

\begin{tabular}{|c|c|c|c|c|c|c|c|c|c|c|}
\hline \multicolumn{11}{|c|}{ DNN } \\
\hline \multicolumn{11}{|c|}{ Medium condition } \\
\hline (Unit: \%) & Lev. 1 & Lev. 2 & Lev. 3 & Lev. 4 & Lev. 5 & Lev. 6 & Lev. 7 & Lev. 8 & Lev. 9 & Lev. 10 \\
\hline Lev. 1 & 90 & 10 & 0 & 0 & 0 & 0 & 0 & 0 & 0 & 0 \\
\hline Lev. 2 & 10 & 90 & 0 & 0 & 0 & 0 & 0 & 0 & 0 & 0 \\
\hline Lev. 3 & 0 & 0 & 95 & 5 & 0 & 0 & 0 & 0 & 0 & 0 \\
\hline Lev. 4 & 5 & 0 & 0 & 95 & 0 & 0 & 0 & 0 & 0 & 0 \\
\hline Lev. 5 & 0 & 0 & 0 & 5 & 95 & 0 & 0 & 0 & 0 & 0 \\
\hline Lev. 6 & 0 & 0 & 0 & 0 & 0 & 100 & 0 & 0 & 0 & 0 \\
\hline Lev. 7 & 0 & 0 & 0 & 0 & 0 & 0 & 100 & 0 & 0 & 0 \\
\hline Lev. 8 & 0 & 0 & 0 & 0 & 0 & 0 & 0 & 95 & 0 & 5 \\
\hline Lev. 9 & 0 & 0 & 0 & 0 & 0 & 0 & 0 & 0 & 95 & 5 \\
\hline Lev. 10 & 0 & 0 & 0 & 0 & 0 & 0 & 0 & 0 & 0 & 100 \\
\hline \multicolumn{11}{|c|}{ Rough condition } \\
\hline (Unit: \%) & Lev. 1 & Lev. 2 & Lev. 3 & Lev. 4 & Lev. 5 & Lev. 6 & Lev. 7 & Lev. 8 & Lev. 9 & Lev. 10 \\
\hline Lev. 1 & 70 & 20 & 5 & 5 & 0 & 0 & 0 & 0 & 0 & 0 \\
\hline Lev. 2 & 15 & 80 & 5 & 0 & 0 & 0 & 0 & 0 & 0 & 0 \\
\hline Lev. 3 & 5 & 0 & 90 & 5 & 0 & 0 & 0 & 0 & 0 & 0 \\
\hline Lev. 4 & 10 & 0 & 5 & 85 & 0 & 0 & 0 & 0 & 0 & 0 \\
\hline Lev. 5 & 0 & 0 & 0 & 10 & 90 & 0 & 0 & 0 & 0 & 0 \\
\hline Lev. 6 & 0 & 0 & 0 & 0 & 5 & 95 & 0 & 0 & 0 & 0 \\
\hline Lev. 7 & 0 & 0 & 0 & 0 & 0 & 0 & 100 & 0 & 0 & 0 \\
\hline Lev. 8 & 0 & 0 & 0 & 0 & 0 & 0 & 0 & 95 & 0 & 5 \\
\hline Lev. 9 & 0 & 0 & 0 & 0 & 0 & 0 & 0 & 0 & 90 & 10 \\
\hline Lev. 10 & 0 & 0 & 0 & 0 & 0 & 0 & 0 & 0 & 0 & 100 \\
\hline
\end{tabular}

Table A3. Confusion matrices for the MLP model in three surface conditions.

\begin{tabular}{|c|c|c|c|c|c|c|c|c|c|c|}
\hline \multicolumn{11}{|c|}{ MLP } \\
\hline \multicolumn{11}{|c|}{ Smooth condition } \\
\hline (Unit: \%) & Lev. 1 & Lev. 2 & Lev. 3 & Lev. 4 & Lev. 5 & Lev. 6 & Lev. 7 & Lev. 8 & Lev. 9 & Lev. 10 \\
\hline Lev. 1 & 85 & 15 & 0 & 0 & 0 & 0 & 0 & 0 & 0 & 0 \\
\hline Lev. 2 & 5 & 90 & 0 & 5 & 0 & 0 & 0 & 0 & 0 & 0 \\
\hline Lev. 3 & 5 & 0 & 95 & 0 & 0 & 0 & 0 & 0 & 0 & 0 \\
\hline Lev. 4 & 0 & 0 & 0 & 95 & 0 & 0 & 5 & 0 & 0 & 0 \\
\hline Lev. 5 & 0 & 0 & 0 & 0 & 100 & 0 & 0 & 0 & 0 & 0 \\
\hline Lev. 6 & 0 & 0 & 0 & 0 & 0 & 100 & 0 & 0 & 0 & 0 \\
\hline Lev. 7 & 0 & 0 & 0 & 0 & 0 & 0 & 100 & 0 & 0 & 0 \\
\hline Lev. 8 & 0 & 0 & 0 & 0 & 0 & 0 & 0 & 100 & 0 & 0 \\
\hline Lev. 9 & 0 & 0 & 0 & 0 & 0 & 0 & 0 & 0 & 100 & 0 \\
\hline Lev. 10 & 0 & 0 & 0 & 0 & 0 & 0 & 0 & 0 & 5 & 95 \\
\hline \multicolumn{11}{|c|}{ Medium condition } \\
\hline (Unit: \%) & Lev. 1 & Lev. 2 & Lev. 3 & Lev. 4 & Lev. 5 & Lev. 6 & Lev. 7 & Lev. 8 & Lev. 9 & Lev. 10 \\
\hline Lev. 1 & 80 & 15 & 5 & 0 & 0 & 0 & 0 & 0 & 0 & 0 \\
\hline Lev. 2 & 15 & 80 & 0 & 5 & 0 & 0 & 0 & 0 & 0 & 0 \\
\hline Lev. 3 & 5 & 0 & 90 & 5 & 0 & 0 & 0 & 0 & 0 & 0 \\
\hline Lev. 4 & 0 & 0 & 0 & 95 & 0 & 0 & 5 & 0 & 0 & 0 \\
\hline Lev. 5 & 0 & 0 & 0 & 5 & 95 & 0 & 0 & 0 & 0 & 0 \\
\hline Lev. 6 & 0 & 0 & 0 & 0 & 0 & 100 & 0 & 0 & 0 & 0 \\
\hline Lev. 7 & 0 & 0 & 0 & 0 & 0 & 0 & 95 & 0 & 0 & 5 \\
\hline Lev. 8 & 0 & 0 & 0 & 0 & 0 & 0 & 0 & 95 & 0 & 5 \\
\hline Lev. 9 & 0 & 0 & 0 & 0 & 0 & 0 & 0 & 0 & 100 & 0 \\
\hline Lev. 10 & 0 & 0 & 0 & 0 & 0 & 0 & 5 & 0 & 5 & 90 \\
\hline
\end{tabular}


Table A3. Cont.

\begin{tabular}{|c|c|c|c|c|c|c|c|c|c|c|}
\hline \multicolumn{11}{|c|}{ MLP } \\
\hline \multicolumn{11}{|c|}{ Rough condition } \\
\hline (Unit: \%) & Lev. 1 & Lev. 2 & Lev. 3 & Lev. 4 & Lev. 5 & Lev. 6 & Lev. 7 & Lev. 8 & Lev. 9 & Lev. 10 \\
\hline Lev. 1 & 60 & 25 & 5 & 10 & 0 & 0 & 0 & 0 & 0 & 0 \\
\hline Lev. 2 & 15 & 70 & 5 & 5 & 0 & 0 & 5 & 0 & 0 & 0 \\
\hline Lev. 3 & 5 & 0 & 85 & 10 & 0 & 0 & 0 & 0 & 0 & 0 \\
\hline Lev. 4 & 10 & 0 & 10 & 75 & 0 & 0 & 5 & 0 & 0 & 0 \\
\hline Lev. 5 & 0 & 0 & 0 & 10 & 85 & 5 & 0 & 0 & 0 & 0 \\
\hline Lev. 6 & 0 & 0 & 0 & 5 & 5 & 85 & 5 & 0 & 0 & 0 \\
\hline Lev. 7 & 0 & 0 & 0 & 0 & 0 & 0 & 95 & 0 & 0 & 5 \\
\hline Lev. 8 & 0 & 0 & 0 & 0 & 0 & 0 & 0 & 90 & 0 & 10 \\
\hline Lev. 9 & 0 & 0 & 0 & 0 & 0 & 5 & 0 & 0 & 80 & 15 \\
\hline Lev. 10 & 0 & 0 & 0 & 0 & 0 & 0 & 5 & 0 & 15 & 80 \\
\hline
\end{tabular}

\section{References}

1. Gorsse, S.; Hutchinson, C.; Gouné, M.; Banerjee, R. Additive manufacturing of metals: A brief review of the characteristic microstructures and properties of steels, Ti-6Al-4V and high-entropy alloys. Sci. Technol. Adv. Mater. 2017, 18, 584-610. [CrossRef] [PubMed]

2. Seifi, M.; Salem, A.; Beuth, J.; Harrysson, O.; Lewandowski, J.J. Overview of Materials Qualification Needs for Metal Additive Manufacturing. JOM 2016, 68, 747-764. [CrossRef]

3. Everton, S.K.; Hirsch, M.; Stravroulakis, P.; Leach, R.K.; Clare, A.T. Review of in-situ process monitoring and in-situ metrology for metal additive manufacturing. Mater. Des. 2016, 95, 431-445. [CrossRef]

4. Babu, B.; Lundbäck, A.; Lindgren, L.-E. Simulation of Ti-6Al-4V Additive Manufacturing Using Coupled Physically Based Flow Stress and Metallurgical Model. Materials 2019, 12, 3844. [CrossRef]

5. Wang, Z.; Palmer, T.A.; Beese, A.M. Effect of processing parameters on microstructure and tensile properties of austenitic stainless steel 304L made by directed energy deposition additive manufacturing. Acta Mater. 2016, 110, 226-235. [CrossRef]

6. Collins, P.C.; Brice, D.A.; Samimi, P.; Ghamarian, I.; Fraser, H.L. Microstructural Control of Additively Manufactured Metallic Materials. Annu. Rev. Mater. Res. 2016, 46, 63-91. [CrossRef]

7. Yang, K.; Xie, H.; Sun, C.; Zhao, X.; Li, F. Influence of Vanadium on the Microstructure of IN718 Alloy by Laser Cladding. Materials 2019, 12, 3839. [CrossRef]

8. Everton, S.; Dickens, P.; Tuck, C.; Dutton, B. Evaluation of laser ultrasonic testing for inspection of metal additive manufacturing. Laser 3D Manuf. II Int. Soc. Opt. Photonics 2015, 9353, 935316. [CrossRef]

9. Clark, D.; Sharples, S.D.; Wright, D.C. Development of online inspection for additive manufacturing products. Insight 2011, 53, 610-613. [CrossRef]

10. Eren, E.; Kurama, S.; Solodov, I. Characterization of porosity and defect imaging in ceramic tile using ultrasonic inspections. Ceram. Int. 2012, 38, 2145-2151. [CrossRef]

11. Honarvar, F.; Varvani-Farahani, A. A review of ultrasonic testing applications in additive manufacturing: Defect evaluation, material characterization, and process control. Ultrasonics 2020, 108, 106227. [CrossRef]

12. Chabot, A.; Laroche, N.; Carcreff, E.; Rauch, M.; Hascoët, J.-Y. Towards defect monitoring for metallic additive manufacturing components using phased array ultrasonic testing. J. Intell. Manuf. 2019, 31, 1191-1201. [CrossRef]

13. Jeong, H.; Hsu, D.K. Experimental analysis of porosity-induced ultrasonic attenuation and velocity change in carbon composites. Ultrasonics 1995, 33, 195-203. [CrossRef]

14. Park, S.-H.; Kim, J.; Jhang, K.-Y. Relative measurement of the acoustic nonlinearity parameter using laser detection of an ultrasonic wave. Int. J. Precis. Eng. Manuf. 2017, 18, 1347-1352. [CrossRef]

15. Jeong, H. Effects of Voids on the Mechanical Strength and Ultrasonic Attenuation of Laminated Composites. J. Compos. Mater. 1997, 31, 276-292. [CrossRef]

16. Kim, H.S.; Bush, M.B. The effects of grain size and porosity on the elastic modulus of nanocrystalline materials. Nanostruct. Mater. 1999, 11, 361-367. [CrossRef]

17. Birt, E.A.; Smith, R.A. A review of NDE methods for porosity measurement in fibre-reinforced polymer composites. Insight 2004, 46, 681-686. [CrossRef]

18. Hernandez, M.G.; Izquierdo, M.A.G.; Ibanez, A.; Anaya, J.J.; Ullate, L.G. Porosity estimation of concrete by ultrasonic NDE. Ultrasonics 2000, 38, 531-533. [CrossRef]

19. Slotwinski, J.A.; Garboczi, E.J.; Hebenstreit, K.M. Porosity Measurements and Analysis for Metal Additive Manufacturing Process Control. J. Res. Natl. Inst. Stand. Technol. 2014, 119, 494-528. [CrossRef]

20. Karthik, N.V.; Gu, H.; Pal, D.; Starr, T.; Stucker, B. High frequency ultrasonic non destructive evaluation of additively manufactured components. In Proceedings of the 24th International Solid Freeform Fabrication Symposium, Austin, TX, USA, 12-14 August 2013; pp. 311-325. 
21. Javidrad, H.R.; Salemi, S. Determination of elastic constants of additive manufactured Inconel 625 specimens using an ultrasonic technique. Int. J. Adv. Manuf. Technol. 2020, 107, 4597-4607. [CrossRef]

22. Bakre, C.; Hassanian, M.; Lissenden, C. Influence of surface roughness from additive manufacturing on laser ultrasonics measurements. In AIP Conference Proceedings; AIP Publishing LLC: Melville, NY, USA, 2019; p. 020009.

23. Calignano, F.; Manfredi, D.; Ambrosio, E.P.; Iuliano, L.; Fino, P. Influence of process parameters on surface roughness of aluminum parts produced by DMLS. Int. J. Adv. Manuf. Technol. 2013, 67, 2743-2751. [CrossRef]

24. Tyagi, P.; Goulet, T.; Riso, C.; Stephenson, R.; Chuenprateep, N.; Schlitzer, J.; Benton, C.; Garcia-Moreno, F. Reducing the roughness of internal surface of an additive manufacturing produced 316 steel component by chempolishing and electropolishing. Addit. Manuf. 2019, 25, 32-38. [CrossRef]

25. Whip, B.; Sheridan, L.; Gockel, J. The effect of primary processing parameters on surface roughness in laser powder bed additive manufacturing. Int. J. Adv. Manuf. Technol. 2019, 103, 4411-4422. [CrossRef]

26. Richardson, F.; Reynolds, D.; Dehak, N. Deep Neural Network Approaches to Speaker and Language Recognition. IEEE Signal Process. Lett. 2015, 22, 1671-1675. [CrossRef]

27. Noda, K.; Yamaguchi, Y.; Nakadai, K.; Okuno, H.G.; Ogata, T. Audio-visual speech recognition using deep learning. Appl. Intell. 2015, 42, 722-737. [CrossRef]

28. Hamel, P.; Eck, D. Learning features from music audio with deep belief networks. ISMIR 2010, 10, 339-344.

29. Kim, Y.; Lee, H.; Provost, E.M. Deep learning for robust feature generation in audiovisual emotion recognition. In Proceedings of the IEEE International Conference on Acoustics, Speech and Signal Processing, Vancouver, BC, Canada, 26-31 May 2013; pp. 3687-3691.

30. Hou, W.; Wei, Y.; Guo, J.; Jin, Y.; Zhu, C. Automatic Detection of Welding Defects using Deep Neural Network. J. Phys. Conf. Ser. 2018, 933, 012006. [CrossRef]

31. Meng, M.; Chua, Y.J.; Wouterson, E.; Ong, C.P.K. Ultrasonic signal classification and imaging system for composite materials via deep convolutional neural networks. Neurocomputing 2017, 257, 128-135. [CrossRef]

32. Zhu, P.; Cheng, Y.; Banerjee, P.; Tamburrino, A.; Deng, Y. A novel machine learning model for eddy current testing with uncertainty. NDT E Int. 2019, 101, 104-112. [CrossRef]

33. Aldrin, J.C.; Forsyth, D.S. Demonstration of using signal feature extraction and deep learning neural networks with ultrasonic data for detecting challenging discontinuities in composite panels. AIP Conf. Proc. 2019, 2102, 020012.

34. Munir, N.; Kim, H.-J.; Park, J.; Song, S.-J.; Kang, S.-S. Convolutional neural network for ultrasonic weldment flaw classification in noisy conditions. Ultrasonics 2019, 94, 74-81. [CrossRef] [PubMed]

35. Gao, F.; Li, B.; Chen, L.; Wei, X.; Shang, Z.; He, C. Ultrasonic signal denoising based on autoencoder. Rev. Sci. Instrum. 2020, 91, 045104. [CrossRef] [PubMed]

36. Margrave, F.; Rigas, K.; Bradley, D.; Barrowcliffe, P. The use of neural networks in ultrasonic flaw detection. Measurement 1999, 25, 143-154. [CrossRef]

37. Yuan, S.; Wang, L.; Peng, G. Neural network method based on a new damage signature for structural health monitoring. Thin-Walled Struct. 2005, 43, 553-563. [CrossRef]

38. Fahad, M.; Kamal, K.; Zafar, T.; Qayyum, R.; Tariq, S.; Khan, K. Corrosion detection in industrial pipes using guided acoustics and radial basis function neural network. In Proceedings of the 2017 International Conference on Robotics and Automation Sciences (ICRAS), Hong Kong, China, 26-29 August 2017; pp. 129-133.

39. Cai, W.; Wang, J.; Zhou, Q.; Yang, Y.; Jiang, P. Equipment and Machine Learning in Welding Monitoring: A Short Review. In Proceedings of the 5th International Conference on Mechatronics and Robotics Engineering, Rome, Italy, 16-19 February 2019; pp. 9-15.

40. Wang, Y.; Shi, F.; Tong, X. A Welding Defect Identification Approach in X-ray Images Based on Deep Convolutional Neural Networks. In Proceedings of the International Conference on Intelligent Computing, Nanchang, China, 3-6 August 2019; pp. 953-964.

41. Isamail, L.; Maskuri, N.L.; Isip, N.J.; Lokman, S.F.; Abu Bakar, M.H. Deep Neural Network Modeling for Metallic Component Defects Using the Finite Element Model. In Progress in Engineering Technology; Springer: Cham, Switzerland, 2019 ; pp. 259-270.

42. Zhang, W.; Li, C.; Peng, G.; Chen, Y.; Zhang, Z. A deep convolutional neural network with new training methods for bearing fault diagnosis under noisy environment and different working load. Mech. Syst. Signal Process. 2018, 100, 439-453. [CrossRef]

43. Grasso, M.; Colosimo, B.M. Process defects and in situ monitoring methods in metal powder bed fusion: A review. Meas. Sci. Technol. 2017, 28, 044005. [CrossRef]

44. Qi, T.; Zhu, H.; Zhang, H.; Yin, J.; Ke, L.; Zeng, X. Selective laser melting of Al7050 powder: Melting mode transition and comparison of the characteristics between the keyhole and conduction mode. Mater. Des. 2017, 135, 257-266. [CrossRef]

45. Guo, Q.; Zhao, C.; Qu, M.; Xiong, L.; Escano, L.I.; Hojjatzadeh, S.M.H.; Parab, N.D.; Fezzaa, K.; Everhart, W.; Sun, T.; et al. In-situ characterization and quantification of melt pool variation under constant input energy density in laser powder bed fusion additive manufacturing process. Addit. Manuf. 2019, 28, 600-609. [CrossRef]

46. Leung, C.L.A.; Marussi, S.; Atwood, R.C.; Towrie, M.; Withers, P.J.; Lee, P.D. In situ X-ray imaging of defect and molten pool dynamics in laser additive manufacturing. Nat. Commun. 2018, 9, 1355. [CrossRef] 
47. Jung, K.-H.; Kim, D.-H.; Kim, H.-J.; Park, S.-H.; Jhang, K.-Y.; Kim, H.-S. Finite element analysis of a low-velocity impact test for glass fiber-reinforced polypropylene composites considering mixed-mode interlaminar fracture toughness. Compos. Struct. 2017, 160, 446-456. [CrossRef]

48. Ziółkowski, G.; Chlebus, E.; Szymczyk, P.; Kurzac, J. Application of X-ray CT method for discontinuity and porosity detection in 316L stainless steel parts produced with SLM technology. Arch. Civ. Mech. Eng. 2014, 14, 608-614. [CrossRef]

49. Koester, L.W.; Taheri, H.; Bigelow, T.A.; Collins, P.C.; Bonds, L.J. Nondestructive Testing for Metal Parts Fabricated Using Powder-Based Additive Manufacturing. Mater. Eval. 2018, 76, 514-524.

50. Jhang, K.-Y.; Choi, S.; Kim, J. Measurement of Nonlinear Ultrasonic Parameters from Higher Harmonics. In Measurement of Nonlinear Ultrasonic Characteristics; Springer: Singapore, 2020; pp. 9-60.

51. LeCun, Y.; Bengio, Y.; Hinton, G. Deep learning. Nature 2015, 521, 436-444. [CrossRef]

52. Hinton, G.E.; Osindero, S.; Teh, Y.-W. A Fast Learning Algorithm for Deep Belief Nets. Neural Comput. 2006, 18, 1527-1554. [CrossRef] [PubMed]

53. Sambath, S.; Nagaraj, P.; Selvakumar, N.; Arunachalam, S.; Page, T. Automatic detection of defects in ultrasonic testing using artificial neural network. Int. J. Microstruct. Mater. Prop. 2010, 5, 561. [CrossRef]

54. LeCun, Y.; Bengio, Y. Convolutional Networks for Images, Speech, and Time-Series. Handb. Brain Theory Neural Netw. 1995, 3361, 1995.

55. Aghdam, H.H.; Heravi, E.J. Guide to Convolutional Neural Networks; Springer: New York, NY, USA, 2017; Volume 10, pp. 973-978.

56. Wang, W.; Zhu, M.; Wang, J.; Zeng, X.; Yang, Z. End-to-end encrypted traffic classification with one-dimensional convolution neural networks. In Proceedings of the IEEE International Conference on Intelligence and Security Informatics (ISI), Beijing, China, 22-24 July 2017; pp. 43-48. [CrossRef]

57. Dahl, G.E.; Sainath, T.N.; Hinton, G.E. Improving deep neural networks for LVCSR using rectified linear units and dropout. In Proceedings of the 2013 IEEE International Conference on Acoustics, Speech and Signal Processing, Vancouver, BC, Canada, 26-31 May 2013; pp. 8609-8613.

58. Srivastava, N.; Hinton, G.; Krizhevsky, A.; Sutskever, I.; Salakhutdinov, R. Dropout: A simple way to prevent neural networks from overfitting. J. Mach. Learn. Res. 2014, 15, 1929-1958.

59. Zhang, C.; Bengio, S.; Hardt, M.; Recht, B.; Vinyals, O. Understanding deep learning requires rethinking generalization. arXiv 2016, arXiv:1611.03530. 